\title{
Statisitical Time Series Decomposition into Significant Components and Application to European Temperature*
}

\author{
Jürgen Grieser ${ }^{\dagger} \quad$ Silke Trömel ${ }^{\ddagger} \quad$ Christian-D. Schönwiese ${ }^{\S}$ \\ J.W. Goethe University, \\ Department of Meteorology and Geophysics, \\ P.O. Box 1119 32, \\ D- 60054 Frankfurt a.M., FRG
}

*with 7 figures

${ }^{\dagger}$ Grieser@meteor.uni-frankfurt.de

‡S.Troemel@meteor.uni-frankfurt.de

§Schoenwiese@meteor.uni-frankfurt.de 


\begin{abstract}
Time series of observed monthly mean temperatures of European stations and at grid points are decomposed into different kinds of trend (linear, progressive, degressive), constant or significantly changing annual cycle,episodic and harmonic components, extreme events and noise. A stepwiseregression is used to test whether the components are significant. Specialemphasis is given to extreme events which we distinguish from extreme values. While extreme values may likely occur by chance, it is very unlikelythat extreme events would be in accordance with the features of the time series. On one hand, extreme events alter the estimates (and test results)of trends and other components. On the other hand, such components have to beknown to recognize extreme events. To deal with this problem, an iterativeprocedure is introduced that converges fast to robust estimates of all thecomponents.

Applying this procedure to European temperatures of the last 100 years can reveal that the phase of the annual cycle is shifted backward inthe year in western Europe but foreward in the eastern part. In thelatter region, the amplitude of the annual cycle has also increasedsignificantly. Most of the trend components found in the time series arepositive and linear. Nearly all detected extreme events are cold events whichoccured in winter. Their number has significantly grown. Significant harmoniccomponents with a period of 92.3 months (about 7.7 years) are found mainly inthe northern and western part of Europe.
\end{abstract}

\title{
1 Introduction
}

One of the most important tasks of modern climatology is to understand climate variability. The effect of a particular natural or anthropogenic forcing canbe valued only if climate variablity is understood. Thus, great effort isbeing given to the analysis of systematic climate variations as they areobserved at numerous meteorological stations worldwide (Nicholls et al.,1996). In particular temperature data are prefered because they seem to be the most reliable observations. In order to understand the variability of temperature time series, many different strategies have been applied. Some authors searched only for trends and thus assumed the time series to be the sum of a certain (mainly linear) trend plus stationary noise (Schönwiese and Rapp, 1997). Other authors tried to explain temperature variations as a result of instationary noise processes (Gordon, 1991; Koscielny-Bunde et al., 1998; Tsonis et al., 1998). However, authors, who tested both of these possibilities alternatively, showed that the observed trends could mostlikely not be explained by noise processes alone (Bloomfield and Nychka,1992; Bloomfield 1992; Zheng and Basher, 1998). This corresponds to theresults obtained from coupled general circulation models (Gates et al.,1996; Kattenberg et al., 1996). These models suggest a trend in temperaturecaused by enhanced greenhouse gas concentrations.

However, a trend can be seen as a smooth change in the mean value of a stochastic variable. Moreover a time series must not only be the sum of atrend and noise, but may be comprised of many other components which may alsoindicate a climate change. Thus some 
authors prefer to decompose a timeseries by singular spectrum analysis(SSA; Vautard and Ghil, 1989) into orthogonal components (Ghil and Vautard,1991; Mann and Park, 1994; Schlesinger and Ramankutty, 1994; Plaut et al.,1995; Moron et al.,1998). The problem with this approach is that theseorthogonal components must neither show obvious temporal structures nor bephysically explicable (Elsner and Tsonis, 1991; Allen et al., 1992; Tsonisand Elsner, 1992; Elsner and Tsonis, 1994, Schlesinger and Ramankutty, 1994). We suggest to apply a generalized additive model (GAM, see e.g. Vislocky and Fritsch, 1995) consisting of the following components:

- trend (linear, progressive or degressive),

- annual cycle (constant or significantly changing),

- episodic component,

- harmonic components (other than annual),

- extrem events, and

- noise (stationary or nonstationary).

Together with according significance tests the decomposition into such components is of particular interest for solving the following questions:

a Is there a significant trend in the observed records?

$\mathrm{b}$ If there is a trend in the observations, is it a linear, a progressive or a degressive trend?

c Has the annual cycle significantly changed and if so, how has it changed?

d Is there a significant episodic structure in the record?

e Are there significant harmonic components?

f Are there extreme values in the record which are not explainable by the statistical features of the record and thus have to be viewed as specialextreme events?

g If all the significant structures of the record are removed may the residuum be a realization of a stationary process?

Dealing with all these questions within one strategy may allow a complete decomposition of a time series and the evaluationof robust estimates of each component in the presence of the other components which are in fact only asymptotic orthogonal. However, such a decomposition strategy does not need to be successful for any kind of time series. Only $a$ posteriori, if a time series is decomposed and the residuals do notdiffer significantly from 
stationary noise, the results may be reliable.

A method is needed for each of the components to test if it is part of the time series. Furthermore estimates of the components are required. To meet these requirements a flexible strategy has to be used that stepwise takes into account the most important component of the time series. We therefore use astepwise regression (see section 3 or von Storch and Zwiers, 1999).

Special emphasis is placed on extreme events as distinguished from extremes. Every time series has $n$ largest and smallest values, which may be called extremes. In addition to that, extreme events, as we define them here are extreme values that are very unlikely to occur only by chance in the presence of the other time series components.

However, one encounters a dilemma in the decomposition process. If there are extreme events which are not homogeniously distributed within the timeseries, they alter the estimates of a trend or in a worst case suggest atrend where is in fact no trend (or vice versa). Thus extreme events have tobe extracted before trends can be estimated correctly. On theother hand, one has to know the trend and other components in order to detect and extract extreme events. We use an iterative approach to deal with this problem. It is introduced and described in section 3.4.

Applying the time series decomposition to observations from different sites, one obtains a spatial discrimination in areas where a component is and is not visible.

We have restricted our analysis on the variations of observed European monthly mean temperature data. Doing this, we use two sets of time series: stationtime series and grid-point time series. In the following section, the datasetsare introduced. The details of the method of analysis are explained insection 3. The results are presented in section 4 and discussed in section 5 .

\section{Data}

In order to investigate long-term climate variations, we prefer climate time series which cover a period of 100 years. To eliminate the pronounced, but partly chaotic weather phenomena we use only monthly averages. Two types of temperatures are analyzed. First, we use times series from a station network which are most likely not inhomogeneous (Rapp and Schönwiese, 1997). They cover the period from 1891 to 1990. The 41 stations from which these time series are taken are presented in Figure 1a. The stations are not uniformly distributred and thus spatial gaps occur, especially over the Iberian peninsula and in the eastern part of Europe. However, these data are raw station data and thus reveal a wealth of local information. Additionally, a grid-point data set $\left(5^{\circ} \times 5^{\circ}\right)$ of 52 time series (Parker et al., 1994, updated) covering the period from 1899 to 1998 is analyzed. The locations of the grid points are shown in Figure 1b. Moreover, these records are temperature anomalies, i.e. the mean annual cycle is substracted. However, this will not influence the results since temporal changes in the annual cycleare not affected by the averaging procedure. 

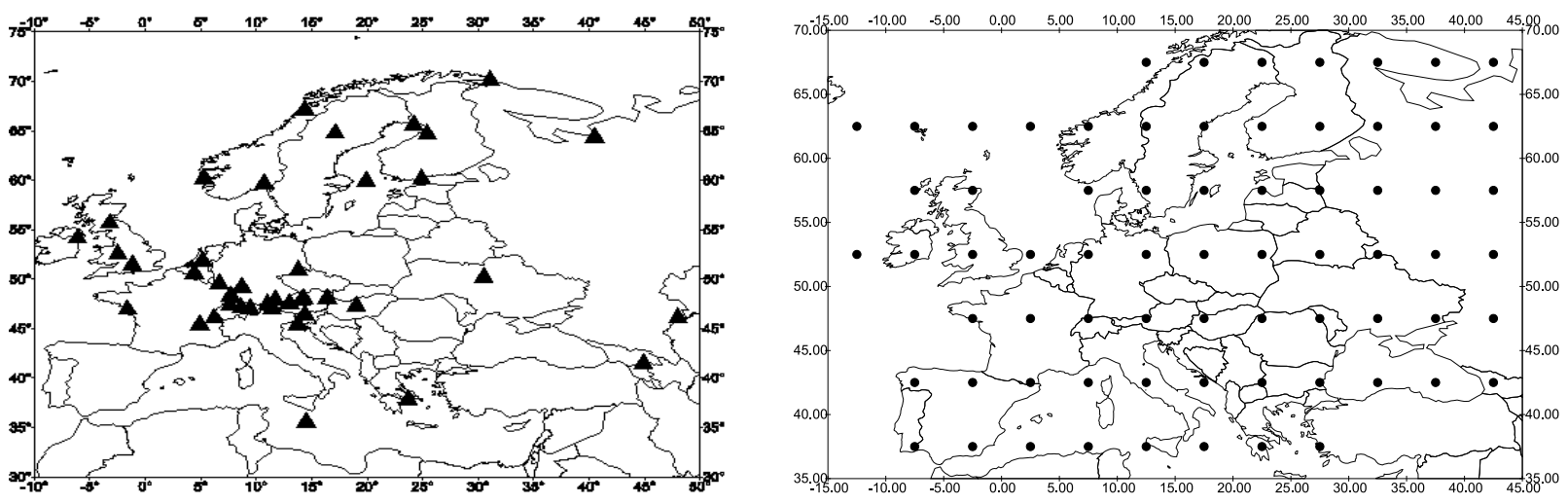

Figure 1: Location of stations (a) with analyzed temperature records (1891-1990) and grid points (b) of analyzed temperature time series (1899-1998).

\section{Method of Analysis}

To decompose temperature time series, we regard them as the following sum of components:

$$
x(t)=m+t_{r}(t)+s(t)+g(t)+h(t)+e(t)+\epsilon(t)
$$

with

$\mathrm{x}(\mathrm{t})=$ original time series,

$\mathrm{m}=$ mean,

$\mathrm{t}_{r}(\mathrm{t})=$ trends,

$\mathrm{s}(\mathrm{t})=$ annual cycle,

$\mathrm{g}(\mathrm{t})=$ episodic component,

$\mathrm{h}(\mathrm{t})=$ harmonic components,

$\mathrm{e}(\mathrm{t})=$ extreme events and

$\epsilon(\mathrm{t})=$ noise.

A flexible strategy is necessary to deal with all these components. We proceed as follows: After the mean is substracted, we search for trends and seasonal variations. Stepwise regression allows the components to compete with one another. Only components which explain a significant amount of the time series variance are extracted. These are the first-guess estimates of the components.

The episodic component is comprised of low-frequency variations. It is subject of the second step in the analysis, because we often found them superposed on trends claiming only a small percentage of variance. Nevertheless, significant structures are eliminated.

Usually, remaining residuals of temperature time series are considered to be the sum of noise and extreme events. These single values, which are not in accordance with the $a$ priori supposed Gaussian noise, are substituted by a normally distributed random variable with the mean and variance of the particular month. Subsequently, we restart the 
decomposition procedure with the modified time series. Using ordinary least squares, this iterative procedure is absolutely necessary because it is sensitive to extreme events. Thus, components retained in the second run differ more or less from the first-guess components. The iterative procedure stops if no further extreme events can be found. Fortunately, the strategy converges fast to robust estimates. Consequently, we needed 3 runs at the most. Decomposition succeeded if the residuals could not be distinguished from a realization of a normally distributed random variable. In this way the, a priori assumed residual distribution is tested after the decomposition. To detect harmonic oscillations, the residuals are also subject to a spectral variance analysis (power spectrum).

The following sections show which functions we use to approximate the various components and describe their detection strategy in more detail.

\subsection{Trend}

We offer trends up to the order 5 as potential regressors to the temperature time series under consideration:

$$
t_{r, i}=a_{i}+b_{i} \cdot t^{i} \quad \text { with } i=1,2, \ldots 5 .
$$

Thus, we are able to detect linear, progressive and degressive trends. But, only functions which can explain a significant amount of variance are taken into account. If only the function of order 1 meets the requirementdefined above, a linear trend is extracted. Trends of higher order describe aprogressive temperature development. Detection of degressive shaped trendscan be realized by superposition of linear and progressive trends, bothexplaining a significant amount of variance.

\subsection{Annual cycle}

Besides fixed annual cycles, changes in amplitude and phase of seasonal variations are possible. The equation

$$
s_{j, k}=c_{j, k}+d_{j, k} t^{k} \cos \left(2 \pi \frac{j}{12} t\right)+e_{j, k} t^{k} \sin \left(2 \pi \frac{j}{12} t\right)
$$

with wave number $\mathrm{j}=1,2, \ldots 6$ per year and $\mathrm{k}=0,1,2$ allows amplitudes with fixed, linear and quadratic time dependancy. Obviously, one time series maysignificantly contain two or three harmonics of the annual cycle with thesame wavenumber $\mathrm{j}$ but different time dependency $\mathrm{k}$ of the amplitude.Superposition yields

$$
s_{j}(t)=A_{j}(t) \cos \left(2 \pi \frac{j}{12}\left(t-t_{j}\right)\right)
$$

with amplitude

$$
A_{j}(t)=\sqrt{\sum_{k=0}^{2}\left(d_{j, k}^{2}+e_{j, k}^{2}\right) t^{2 k}}
$$


and phase

$$
t_{j}(t)=\frac{12}{2 \pi} \arctan \left(\frac{\sum_{k=0}^{2} d_{j, k} t^{k}}{\sum_{k=0}^{2} e_{j, k} t^{k}}\right) .
$$

In the case of 6 wavenumbers, equation 3 represents a pool of 33 potential regressors, which enable us to detect linear, progressive and degressive shaped changes in phase and amplitude of the annual cycle. To be sure that we detect all significant structures and that alternatively unsiginificant structures are neglected, detection of trends and annual cycle is performed simultaneously. To decide which of the 5 trend components and 33seasonal components explain a significant amount of the time series variance,the Pearson correlation coefficient between the considered time series andeach potential regressor is computed. The regressor with the largest significant correlation coefficient is extracted from the time series. As with the original time series, the Pearson correlation coefficients between the potential regressors and the resulting residuals are calculated. When the largest correlation coefficient is significant, another winner is found which joins in a multiple regression with the original time series. Again, we offer the 5 trend and 33 seasonal components to the received residuals. In case of a further winner, a third regressor is joined to the multiple regression. The procedure is terminated if all significant regressors are found.

\subsection{Episodic component}

For the detection of relatively low-frequency variations, we fit polynomial equations up to the order 5:

$$
g_{l}(t)=a_{o}+\sum_{i=1}^{l} a_{i} t^{i} \quad \text { with } l=1,2, \ldots 5 .
$$

The polynomial equation of the highest order $l$, which makes a significantly greater contribution of variance than the polynomial equation of order $l-1$ is chosen for regression. In this way, a maximum of two warm and two comparatively cold periods can be detected and eliminated.

\subsection{Extreme events}

As already mentioned, at this stage of the analysis only a small number of rare extreme values may let the residuum differ from Gaussian noise. They are likely not in accordance with the features of the time series. Suchunexpected values may be observation errors, unlikely events which may occurby chance or special, unlikely events which did not happen by chance. Thelatter are called extreme events in this paper. We regard the value with the greatest distance to the mean as potential extreme event. Subsequently, the probability for the accidental occurence of values is computed. With a small probability the potential extreme event is likely to be not in accordance with the distribution of the time series. That means, that the value is probably considered to be an extreme event. The computation of this probability is shown in the following. The detection criterion is based on the hypothesis 
that the residuals, considered without extreme events, are normally distributed. Starting with the probability density function of a normally distributed time series with mean $\mu=0$ and variance $\sigma^{2}=1$, we yield the event probability of a value $\mathrm{z} \leq \mathrm{Z}$ :

$$
p(z \leq Z)=\frac{1}{\sqrt{2 \pi}} \int_{-\infty}^{Z} \exp \left(-\frac{z^{2}}{2}\right) d z^{\prime} .
$$

Using the symmetry of the probability density function and the error function the probability for a value between $-Z$ and $Z$ can be expressedas

$$
p(-Z \leq z \leq Z)=2 p(z \leq Z)-1=\operatorname{erf}\left(\frac{Z}{\sqrt{2}}\right) .
$$

Thus, $1-\mathrm{p}(-\mathrm{Z} \leq \mathrm{z} \leq \mathrm{Z})$ denotes the probability to meet by chance an absolute value greater than $Z$. Preceding equations refer to a single drawing from a normally distributed random variable. Within the scope of this work, we analyse monthly temperature records of the last 100 years. We furthermore assume that these rare extreme events areindependent. In this case the probability $\tilde{p}$ for drawing $\mathrm{k}$ times a value ocurring with the event probability $1-\mathrm{p}(-\mathrm{Z} \leq \mathrm{z} \leq \mathrm{Z})$ within $\mathrm{N}(=1200)$ realizations follows the binomial distribution. For $\mathrm{N} \geq 100$ and $1-\mathrm{p}(-\mathrm{Z} \leq \mathrm{z} \leq \mathrm{Z}) \leq 0.05$ the binomial distribution can be approximated by the Poisson distribution

$$
\tilde{p}(k, N, p)=\frac{\exp ^{-\lambda} \lambda^{k}}{k !} \quad \text { with } \lambda=N(1-p(-Z \leq z \leq Z)) .
$$

In the case of $k=0$, we retain the probability that we meet by chance no value of the considered amount in a time series compassing $\mathrm{N}$ values. If $\tilde{p} \leq 0.1$, the largest or smallest value is with a probability of atleast $90 \%$ not expected and detected as extreme event. The detection procedure works as follows:

First the value found at the greatest distance of the actual mean is selected as potential extreme event. Subsequently we compute the mean and the variance of the remaining residuals. The reduced series is subject to theKolmogorov-Smirnov test (Press et al., 1992) to examine if it differs significantly from Gaussian noise. With the aid of equation (10) we decide whether the potential extreme event is expected in the time series or not. If not, the detected extreme event is extracted and replaced by a normally distributed random value taken from a Gaussian variable with mean and variance of the related month. If the time series involves several extreme events, the described procedure can be repeated to eliminate them successively. Usually, elimination of extreme events reduces the rejection probability of normal distribution. Sometimes initially rejected residuals conform with the normal distribution only if all extreme events are extracted.

\subsection{Residuals}

If the examined temperature time series can be described as a superposition of the considered components, the residuum represents random noise. Using again the KolmogorovSmirnov test, we determine whether the decomposition is reliable or not. If the residual 
record shows also stationarity of mean,variance and autocorrelation, it can be taken as consequence of manyunimportant influences. Testing the hypothesis of stationarity, the samplingtime is divided into two subintervals, and we estimate mean, variance and autocorrelation up to lag 18 for both. For examination into significant differences between the respective two quantities, a t-test is used for the mean and a F-test for the variance. A Fisher transformation makes the differences between the correlation coefficients converge faster to normal distribution. Subsequently, the latter can be used to test for significant differences (see again Press et al., 1992). With these tests we can find instationarities. However, if we do not find a significant instationarity in either of the features, the residuum cannot be regarded as a realization of a stationary noise process. Still, it does not contradict to this hypothesis.

\subsection{Harmonic component}

To detect harmonic oscillations (in addition to the annual cycle), we calculate the periodogramm (Press et al., 1992), i.e. the spectral densities at certaindefined frequencies. The spectral portion of variance is given by

$$
S(f)=N\left(\left(\frac{1}{N} \sum_{t=1}^{N}\left(x_{t}-\bar{x}\right) \cos (2 \pi f t)\right)^{2}+\left(\frac{1}{N} \sum_{t=1}^{N}\left(x_{t}-\bar{x}\right) \sin (2 \pi f t)\right)^{2}\right)
$$

where $\mathrm{f}$ denotes the Fourier frequency, $\bar{x}$ the mean, $\mathrm{N}$ the length and the time index of the time series. With the aid of the periodogramm, harmonic oscillations embedded in Gaussian white noise can easily be detected. However, both the Anderson-Darling and the Kolmogorov-Smirnov test (see again Press et al., 1992) reject the hypothesis of white noise on a high level for all the time series investigated. This reflects the inertia of the climate. The testing of peaks in the periodogram becomes more difficult in this case. Nevertheless, it is not necessary to test the significance of the largest peak in each of the periodograms. Instead, we draw significant results from the number of time series which have their largest peak in the periodogramm at the same period. In this approach, it is not necessary that either of the largest peaks may be significant.

\section{Results}

In the folowing subsections, the different results are presented and discussed. Since all significance tests are performed with anerror-probability of $10 \%$, a feature that is only found in a small fraction ofcases may not be significant within the ensemble, i.e. it can be expected tofind 4 cases of the 41 records that pass the significance test although the searched for feature is found only by chance. Since this is the problem of Bernoulli trials, we can easily find the fraction of the ensembles which must show a feature to be significant on a given level. For both the ensembles under investigation the results are given in Table 1. 
Table 1: Number $n$ of time-series which have to show a feature on the $90 \%$-significance level to be a significant fraction of the ensemble on a given probability level.

\begin{tabular}{c||cccc}
\hline Number of records & $n(90 \%)$ & $n(95 \%)$ & $n(99 \%)$ & $n(99.99 \%)$ \\
\hline 41 stations & 8 & 8 & 10 & 13 \\
52 grid points & 9 & 10 & 12 & 15 \\
\hline
\end{tabular}

Table 2: Number of trends detected in temperature time series. The abbreviations are as follows: $\mathrm{p}=$ progressive, $\mathrm{l}=$ linear, $\mathrm{d}=$ degressive.

\begin{tabular}{|c|c|c|c|c|c|}
\hline \multirow[b]{2}{*}{ kind } & \multirow{2}{*}{$\begin{array}{l}\text { negative } \\
\begin{array}{ccc}\mathrm{p} & \mathrm{l} & \mathrm{d}\end{array}\end{array}$} & \multirow[t]{2}{*}{ none } & \multicolumn{3}{|c|}{ positive } \\
\hline & & & d & 1 & $\mathrm{p}$ \\
\hline stat & 1 & 6 & 2 & 29 & 3 \\
\hline grid points & 5 & 5 & 4 & 24 & 14 \\
\hline
\end{tabular}

\subsection{Trends}

The distribution of trends of different kind are given in Table 2. Only one of 41 time-series of station temperatures (the station De Bilt in The Netherlands) shows a negative trend. In contrast to this, 34 stations show a positive trend. 29 of them are linear.

The grid-point data set structurally shows the same features. There are 5 significant negative trends which seem to be randomly distributed across Europe (see Figure 2), but the overwhelming majority of 42 grid points shows a positive trend.

Thus, on the basis of both the data sets used, it can be concluded that the temperature trend over Europe is positive and mainly linear.

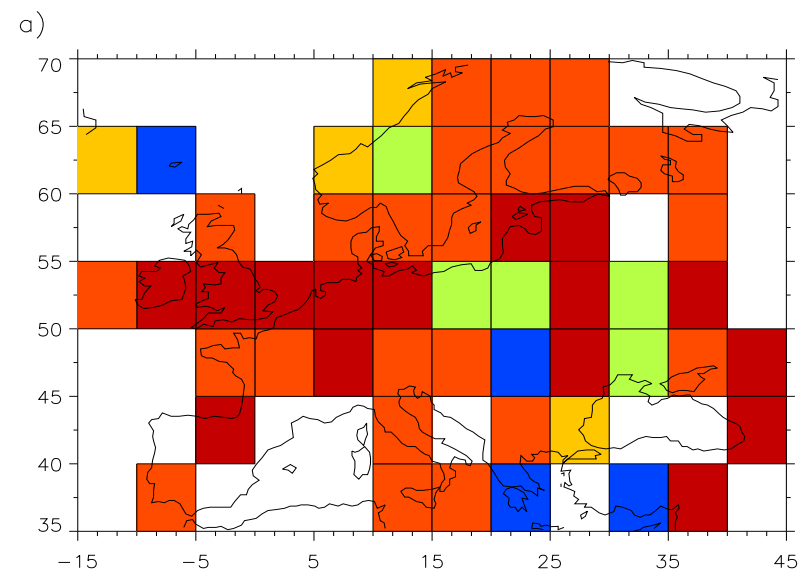

Figure 2: Spatial distribution of trend types in grid-point data of temperature in Europe. 


\subsection{Annual Cycle}

In Table 3, the number of time series with significant changed amplitudes and phase angles of the annual cycle are listed. From that, it can be seen that a significant amount of stations and grid points show changes in the annual cycle. Changes in the amplitudes of the one cycle per year harmonic and the other harmonics are found, indicating a change in the shape of the annual cycle. Significant changes in phase angle are found only in the case of the station data and forthe fundamental frequency.

To get more insight, the number of time series with positive and with negative changes in amplitude and phase angle of the fundamental frequency are subdivided and given in Table 4. It can be seen that a significant part of 9 station records show positive phase angles and exactly the same significant number of stations reveal negative ones. Figure $3 \mathrm{~b}$ shows the spatial distribution of the sign of the change in phase angle of the fundamental frequency. It can be seen that significant positive changes occur mainly in western Europe whereas negative ones appear mainly in the eastern part (exept for the station Kiew). From Figure 3a it can be seen that 6 of 7 stations with a positive change in amplitude of the one cycle per year harmonic are found in the north and north-west of Europe. The significant amount of stations (see Table 4) where the amplitude of the annual cycle is decreasing is found in central and eastern Europe.
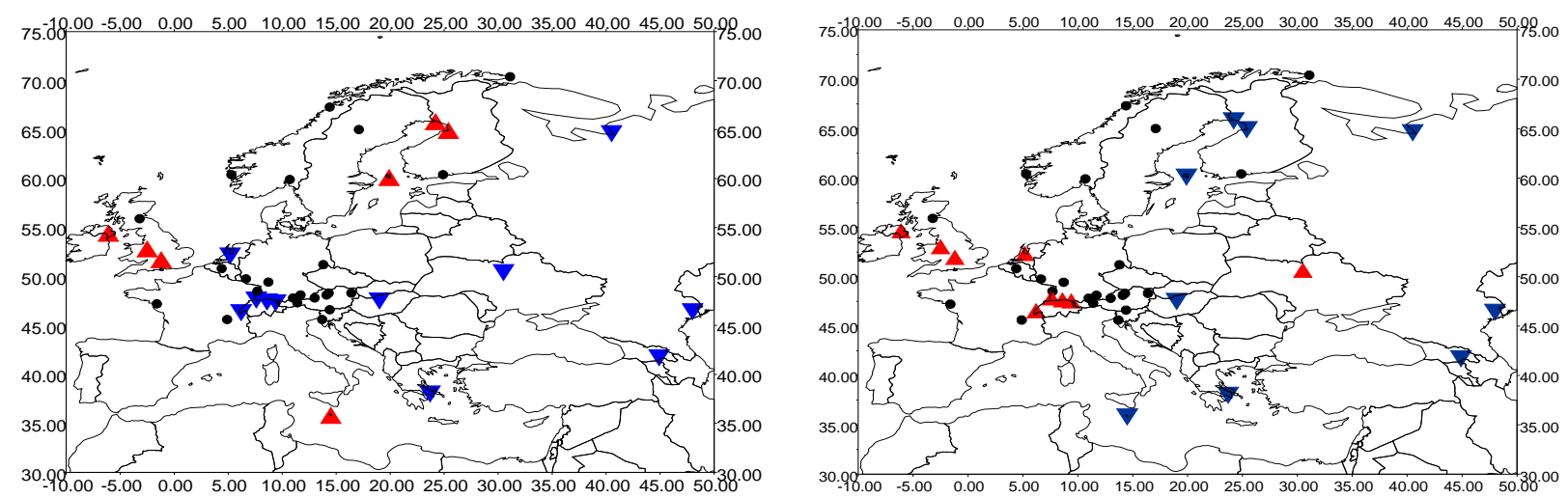

Figure 3: Changes in the annual cycle of temperatures at 41 European stations. a) Changes in the Amplitude of the fundamental period. b) Changes in the phase angle of the fundamental period. Upward showing triangles $=$ positive changes, downward showing triangles $=$ negaitve changes and black spots $=$ no significant changes .

Regarding Figure 4a, a significant number of grid points with increasing amplitudes is also found in eastern Europe. At this point, the results for the gridded data contradict the results for the station data. These contradictions remain when comparing observations with results from general circulation models. Thomson (1995) found the $\log _{2}$ of theatmospheric $\mathrm{CO}_{2}$ levels to be connected with changes in the seasonl cycle.But results from general circulation models are equivocal. Mann (1996) usedthe estimates Northern Hemi- 
sphere average monthly temperature series of Joneset al. with intact seasonl cycle to compare its changes in the seasonal cyclewith responses of the Geophysical Fluid Dynamics Lab (GFDL) coupledocean-atmosphere model and the NCAR Community Climate Model $(\mathrm{CCM})$ general circulation / slap ocean model to increased $\mathrm{CO}_{2}$ concentrations. Observation and model response are similar concerning the decreased amplitude of the annual cycle, but the phase advance found in the observation contradicts thesignificant phase delays in the simulations.
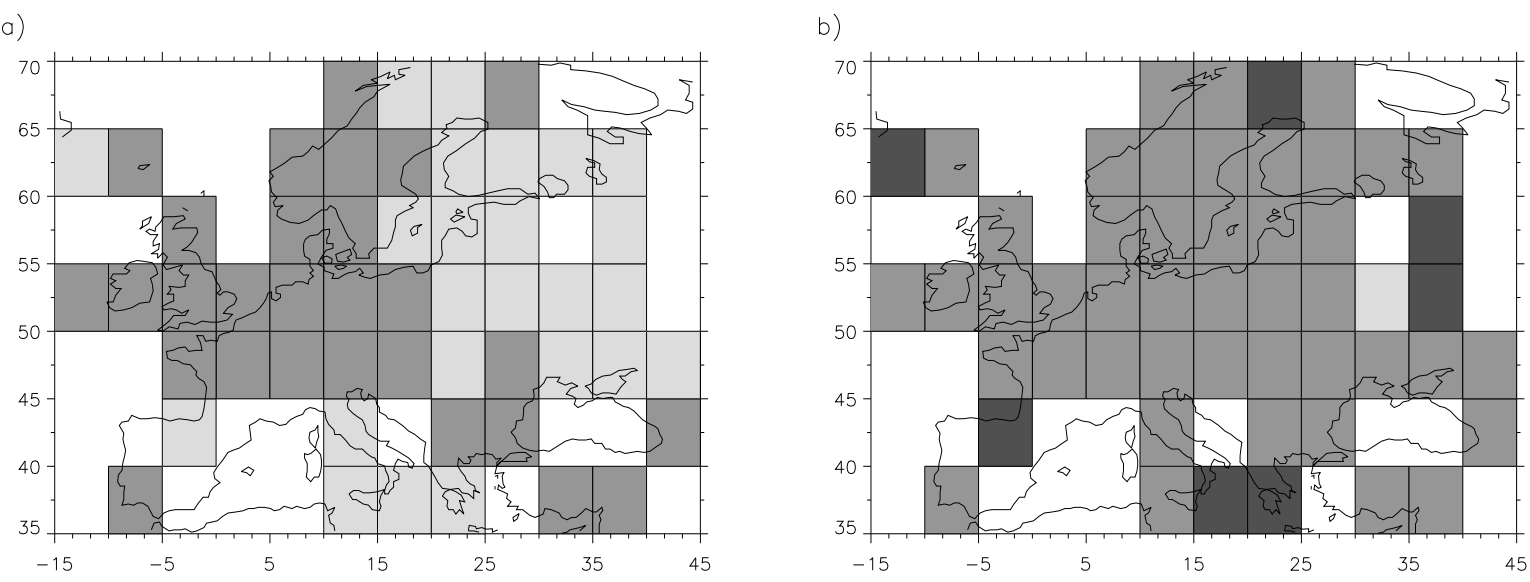

Figure 4: Changes in the annual cycle of temperatures at 52 European grid points. a) Changes in the Amplitude of the fundamental period. b) Changes in the phase angle of the fundamental period. Light grey = positive changes, dark grey $=$ negative, medium grey $=$ no significant changes, and white boxes = lag of data.

Table 3: Number of temperature time series with changed amplitudes $A$ and with changed phase angles $\varphi$ of the annual cycle. Fraction of records in which the amplitude $A$ and the phase angle $\varphi$ of the fundamental frequency have changed as well as the fraction of records with changed phase angle and amplitude of at least one harmonics. Heavy numbers indicate that the $90 \%$ significance level is exceeded.

\begin{tabular}{c||cc|cc|cc}
\hline \multicolumn{1}{c||}{} & \multicolumn{2}{c|}{ Total } & \multicolumn{2}{c|}{ Fundamental } & \multicolumn{2}{c}{ Harmonics } \\
& $A$ & $\varphi$ & $A$ & $\varphi$ & $A$ & $\varphi$ \\
\hline 41 stations & $\mathbf{2 9}$ & $\mathbf{1 9}$ & $\mathbf{1 8}$ & $\mathbf{1 8}$ & $\mathbf{2 4}$ & 3 \\
52 grid points & $\mathbf{3 0}$ & 9 & $\mathbf{2 4}$ & 8 & $\mathbf{1 0}$ & 2 \\
\hline
\end{tabular}

\subsection{Episodic Component}

The order of the polynomials which are fitted as an approximation of the episodic component of the time series are given in Table 5. Innearly all time-series, episodic components are found which explain only aminor fraction of variance. However, to extract extreme 
Table 4: Number of temperature time series with different kinds of changes in the fundamental frequency of the annual cycle. Heavy numbers indicate that the $90 \%$ significance level is exceeded.

\begin{tabular}{c||cc|cc}
\hline & \multicolumn{2}{|c|}{ Changes in amplitude } & \multicolumn{2}{c}{ Changes in phase } \\
& positive & negative & positive & negative \\
\hline 41 stations & 7 & $\mathbf{1 1}$ & $\mathbf{9}$ & $\mathbf{9}$ \\
52 grid points & $\mathbf{2 4}$ & 0 & 1 & 7 \\
\hline
\end{tabular}

events, it isimportant to separate this component as part of thedecomposition strategy. As an example of the spatial homogeneity of thiscomponent, Figure 5 shows the 22 episodic components of the order 5 found in the station data. Although they are found at different locations,they are all very similar especially in the recent part of the interval withone exception at the station of Athens.

Table 5: Number of temperature time series with episodic components of different order and its explained part of variance.

\begin{tabular}{c|ccccc|c}
\hline Order & 0 & 2 & 3 & 4 & 5 & explained variance [\%] \\
\hline \hline 41 stations & 1 & 2 & 2 & 14 & 22 & 1.2 \\
52 grid points & 3 & 0 & 12 & 23 & 14 & 2.5 \\
\hline
\end{tabular}

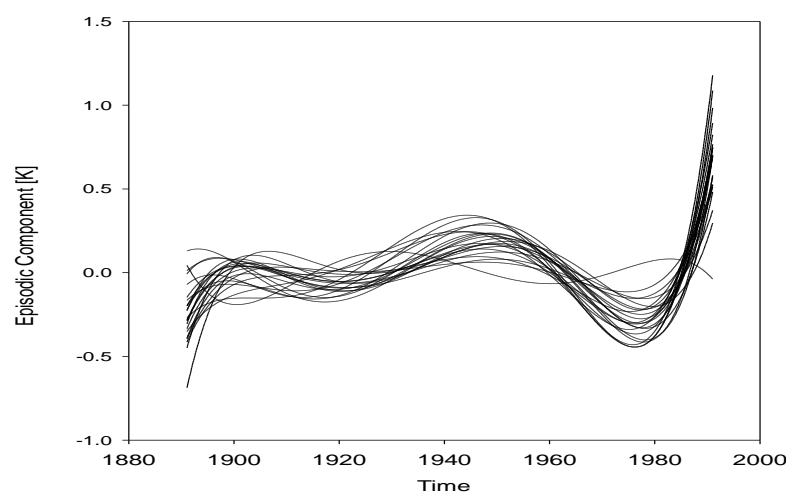

Figure 5: Polynomials of the order 5 as they reflect the episodic component of 22 station records of temperature over Europe. Only 1 station (Athens) shows a pronounced difference in the recent years.

\subsection{Extreme Events}

The number of detected extreme events (total, cold events and warm events) in both the data sets are presented in Table 6 for all seasons and subdivided into the first and second 
half of the records. The strategy applied to detect extreme events shows that 207 out of 49,200 values in the station data set and 228 out of 62,400 values in the grid-point data set areextreme events. These extreme events are not arbitrarily distributed. On thecontrary, from Table 6 it can be seen that a tremendous majorityof extreme events (194 of 207 in case of station data and 158 of 228 in caseof grid-point data) are cold winter events. The 8 warm events within thestation data are not enough to supply any meaningfull information. Thenumber of cold events within the station data has substantially increasedfrom 71 in the period 1891 - 1940 to 123 in the period $1941-1990$.

Although the grid-point data show also a majority of winter cold events, there are two important differences compared to the station data. First, nearly one fifth (41 of 228) of the extreme events are warm events, and second, there are considerably more extreme events detected in the first half as compared to the second one.

This is in contrast to the results of the station data set and may be due to the averaging procedure. In the recent half of the time series, there are more station data available to formulate the grid-point data set. Thus, on the one hand, more regional extremes are smoothed out by the procedure and become invisible. On the other hand, the larger variability at the beginning of theaveraged time series leads to some values that are too warm compared to theoverall standard deviation. Thus, these values are detected as warm eventswhich may mainly be a result of the availability of data and the averagingprocedure.

Table 6: Number and kind of extreme events within first and second half of the different data sets. If the difference between both the halves is significant, the numbers are given in bold. Cold $=$ cold events, Warm $=$ warm events.

\begin{tabular}{c||ccc|ccc|ccc}
\hline \multicolumn{1}{c||}{} & \multicolumn{3}{c|}{ Total Period } & \multicolumn{3}{c|}{ First Half } & \multicolumn{3}{c}{ Second Half } \\
41 Stations & Total & Warm & Cold & Total & Warm & Cold & Total & Warm & Cold \\
\hline Spring & 4 & 2 & 2 & 2 & 2 & 0 & 2 & 0 & 2 \\
Summer & 1 & 0 & 1 & 1 & 0 & 1 & 0 & 0 & 0 \\
Autumn & 4 & 2 & 2 & 2 & 1 & 1 & 2 & 1 & 1 \\
Winter & 198 & 4 & 194 & $\mathbf{7 1}$ & $\mathbf{0}$ & $\mathbf{7 1}$ & $\mathbf{1 2 7}$ & $\mathbf{4}$ & $\mathbf{1 2 3}$ \\
year & 207 & 8 & 199 & $\mathbf{7 6}$ & 3 & $\mathbf{7 3}$ & $\mathbf{1 3 1}$ & 5 & $\mathbf{1 2 6}$ \\
\hline 52 Grid points & Total & Warm & Cold & Total & Warm & Cold & Total & Warm & Cold \\
\hline Spring & 18 & 6 & 12 & $\mathbf{1 6}$ & 5 & $\mathbf{1 1}$ & $\mathbf{2}$ & 1 & $\mathbf{1}$ \\
Summer & 7 & 6 & 1 & 5 & 4 & 1 & 2 & 2 & 0 \\
Autumn & 21 & 5 & 16 & 13 & $\mathbf{5}$ & 8 & 8 & $\mathbf{0}$ & 8 \\
Winter & 182 & 24 & 158 & $\mathbf{1 1 2}$ & $\mathbf{1 8}$ & $\mathbf{9 4}$ & $\mathbf{7 0}$ & $\mathbf{6}$ & $\mathbf{6 4}$ \\
year & 228 & 41 & 187 & $\mathbf{1 4 6}$ & $\mathbf{3 2}$ & $\mathbf{1 1 4}$ & $\mathbf{8 2}$ & $\mathbf{9}$ & $\mathbf{7 3}$ \\
\hline
\end{tabular}

To illustrate the necessity to separate extreme events from the usual values, Figure 6 shows the frequency distributions of the temperature records of Munich with the original data including the annual cycle, the eliminated trend and annual cycle, and after the separation of the extreme events. While the first case of the observed distribution is a convolution of the U-shaped distribution from the annual cycle, the rectangular distribution of the 
linear trend, and the distribution of the residuum and the extreme events, the distribution is more like a Gaussian one after the elimination of trend and annual cycle. However, it also differs considerably from the Gaussian distribution because of some extreme events. After the elimination of these events, the residual distribution is not discernible from the Gaussian distribution.

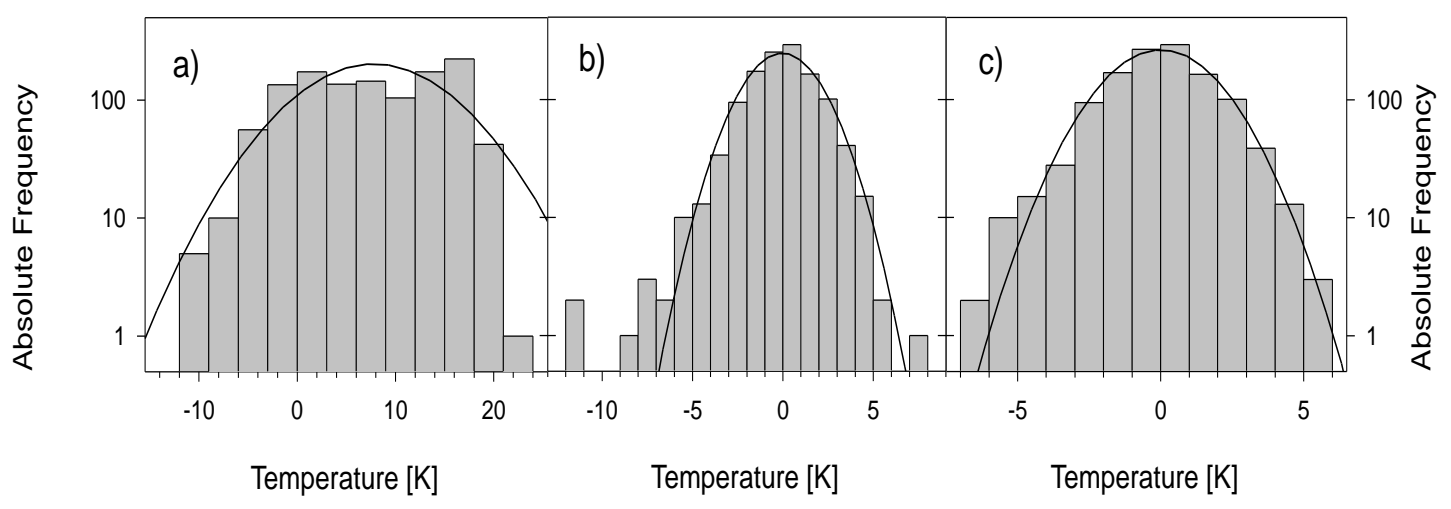

Figure 6: Frequency distribution of the temperature record of Munich (1891-1990). a) Original observations, b) after elimination of first estimates of trend and annual cycle and c) after supplementary elimination of extreme events. All plots are given in a logarithmic scale to let the extreme events become more visible. For comparison the best-fit Gaussian distributions are plotted, too.

As Figure 7 shows the extreme cold winter events are not randomly distributed over Europe. On the contrary, most cold events are found at and south of the Baltic Sea. Since this region marks a transition between thecold continental and the mild maritime winter conditions, it reflects the factthat in some winters continental conditions also dominate in the central partof Europe.

\subsection{Residuals}

The results from searching for instationarities and deviations from a Gaussian distribution are given in Table 7. For comparison this part of the analysis is also applied to the original 

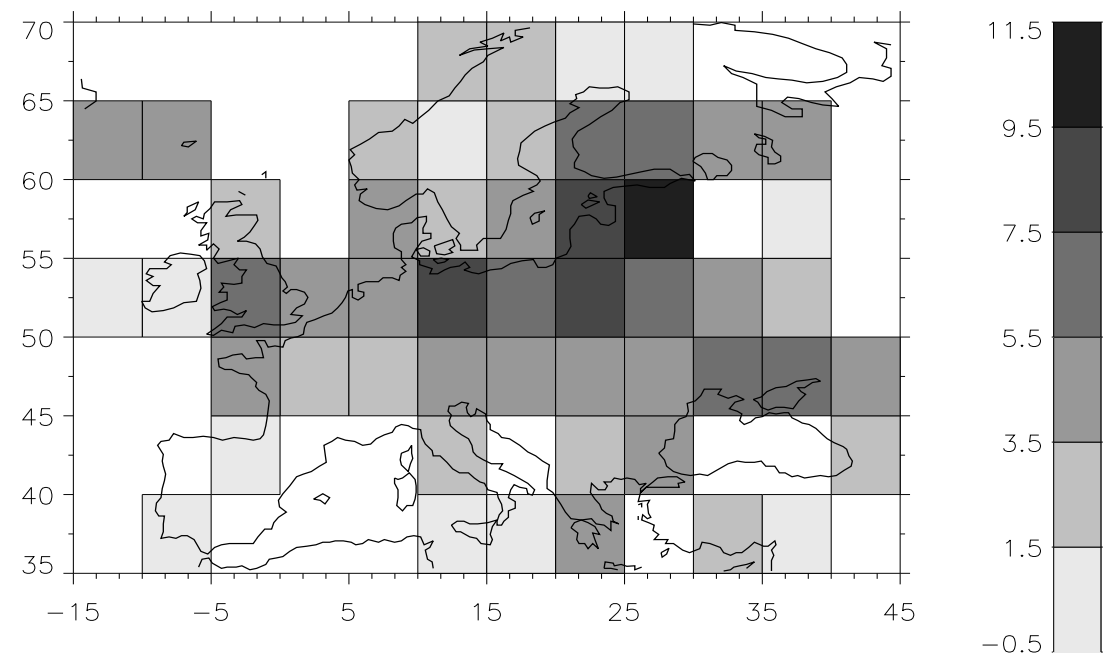

Figure 7: Number of extreme cold events in Europe (1896 - 1995).

records.

Since the 41 records from the temperature stations contain their pronounced annual cycle, all of them differ from the Gaussian distribution and nosignificant instationarity is visible. After eliminating all the significant components no significant amount of stations differ from the assumption of Gaussian distribution and only instationarities in the estimated autocorrelation are found.

The analysis of the 52 records of the gridded data reveals significant instationarities. A significant amount of the original records show an increase in mean and a decrease in variance. The latter is also visible after the elimination of the significant components. The increasing means in the gridded data are detected because the gridded data are anomalies without the annual cycle. Thus, a small shift in the means easily visible than in the station records. After the decomposition, the increases in means vanishes. However, the decrease in the standard deviations and the instationarities in the autocorrelations remain.

We conclude that both these effects result from the averaging procedure used by Parker et al. (1994) who have taken more stations into account for the recent time than about 100 years before. Thus, the variance of the average time series has to decrease, and autocovariance should be affected.

\subsection{Harmonic Components}

Each of the periodograms of the records consists of 600 spectral densities at 600 Fourier periods between 2 and 600 months. Thus, it is a highly significant result that 18 of 41 
Table 7: Statistical features of the time series before (a) and after (b) elimination of temporal structures. $n_{g}$ is the number of records differing significantly from the Gaussian distribution. $\mu, \sigma^{2}$, and $\gamma$ mark mean, variance and autocorrelation, respectively. inc $=$ number of records with a increasing feature, dec $=$ number of records with a decreasing feature, and const $=$ no significant difference between first and second half detected. inst $=$ instationarity. Heavy numbers indicate that the $90 \%$ significance level is exceeded.

\begin{tabular}{|c|c|c|c|c|c|c|c|c|c|}
\hline & \multirow[t]{2}{*}{$n_{g}$} & \multicolumn{3}{|c|}{$\mu$} & \multicolumn{3}{|c|}{$\overline{\sigma^{2}}$} & \multirow{2}{*}{$\begin{array}{c}\gamma \\
\text { inst }\end{array}$} \\
\hline & & & inc & const & dec & inc & const & dec & \\
\hline \multirow{2}{*}{41 Stations } & (a) & 41 & 1 & 39 & 1 & 2 & 39 & 0 & 0 \\
\hline & (b) & 3 & 0 & 41 & 0 & 1 & 36 & 4 & 9 \\
\hline \multirow{2}{*}{52 Grid points } & (a) & 23 & 34 & 15 & 3 & 4 & 16 & 32 & 19 \\
\hline & (b) & 11 & 0 & 52 & 0 & 3 & 18 & 31 & 17 \\
\hline
\end{tabular}

periodograms of the station data show their largest peak at a period of 92.31 months or to 7.69 years. With 52 grid points, 16 periodograms have their largest peak at exactly the same period. It must be concluded that a periodic component of about 7.7 years is within the data field. The same cyclic component is also found in index records of the North-Atlantic Oscillation (Werner, 1999).

\section{Summary and Discussion}

We start with the assumption that the European temperature time series can be decomposed into a sum of trend, annual cycle, episodic and harmonic components, extreme events, and noise. A strategy is introduced toobtain robust estimates of all these components. The strategy is applied totwo different kinds of time series: station records and grid-point data. While the decomposition of the station data led to a complete decomposition of the temporal structures plus noise, the decomposition of the grid-point data led to residuals which differ considerably from the assumption of stationary noise. This may be a result of the averaging procedure, which takes more stations for the recent part of the time interval into account. This should affect all the components which are altered by the averaging procedure and thus at least the standard deviation, autocorrelation and number of extreme events. From this point of view, it follows that station data and grid-point data contain different information. This should be kept in mind while analyzing and interpreting time series.

However, the station records and the grid-point data have some features in common. It could be shown that the temperature trends over Europe are mainly positive and linear. This result proves that the temperature trends can be viewed as linear, an assumption often used while investigating linear trends alone.

Furthermore, we found significant changes in the annual cycle which are partly in accordance with the results of Thomson (1995). However, with the approach given in this paper, 
a more detailed analysis is possible, which ledto additional results. So, we see positive phase shifts in western and negative ones in eastern Europe. An increase of the amplitudes of the fundamental frequency is mainly seen over eastern Europe.

The strategy also reveals some warmer and some colder episodes, each of which lasted for several decades. Although these components are significant, they explain only a minor part of the overall variance. Nevertheless, small changes in mean value may lead to large changes in the probability of exceeding some threshold values and thus may affect the probability of extreme events.

The search for extreme events as extreme values that are not in accordance with the features of the time-series led to a significant number of extreme cold winter events. Despite of the positive temperature trend, the number of the extreme cold winter events is rising. From this result, it follows that it is important to distinguish between normal winters with rising temperatures and winters with extreme events. Only the distinction between them leads to reliable estimates of both.

The residuals show an harmonic component of about 7.7 years which is also seen in the North-Atlantic Oscillation (Werner, 1999).

All these significant components and the features of the residual noise may now be subject of further research aimed at explaining them. As an example, general circulation models may explain the temperature trend by anthropogenic greenhouse gas emissions.

Additionally, time-series of other variables and other regions should be subject to decomposition in order to identify the temporalstructures and the type contained in these time-series.

\section{Acknowledgements}

We are very grateful to the Federal Environmental Agency, Germany, for the support of this study, especially the interest and advice of Mrs. K. Kartschall.

Furthermore, we thank J. Rapp for providing the station data and Paul Sparwasser for his support in improving our English. 


\section{References}

Allen, M.R., P.L. Read and L.A. Smith, 1992: Temperature Oscillations. Nature, 359, 679. Bloomfield, P., 1992: Trends in Global Temperature. Clim. Change, 21,1- 16.

Bloomfield, P. and D. Nychka, 1992: Climate Spectra and Detecting Climate Change. Clim. Change, 21, $275-287$.

Elsner J.B. and A.A. Tsonis, 1991: Do bidecadal oscillations exist in the global temperature record? Nature, 353, 551 - 553 .

Elsner J.B. and A.A. Tsonis, 1994: Low-frequency oscillation. Nature, 372, 507 - 508.

Gates, W.L., A. Henderson-Sellers, G.J. Boer, C.K. Folland, A. Kitoh, B.J. McAvaney, F. Semazzi, N. Smith, A.J. Weaver and Q.-C. Zeng, 1996: Climate Models - Evaluation, in: Houghton, J.T., L.G. Meira Filho, B.A. Callander, N. Harris, A. Kattenberg and K. Maskell, 1996: Climate Change 1995, The Science of Climate Change. Cambridge: Cambridge University Press, 527 pp.

Ghil, M. and R. Vautard, 1991: Interdecadal oscillations and the warming trend in global temperature time series. Nature, 380, $324-327$.

Gordon, A.H., 1991: Global Warming as a Manifestation of a Random Walk. J. Clim., 4, $589-597$.

Kattenberg, A., F. Giorgi, H. Grassl, G.A. Meehl, J.F.B. Mitchel, R.J. Stouffer, T. Tokioka, A.J. Weaver and T.M.L. Wigley, 1996: Climate Models - Projections of Future Climate, in: Houghton, J.T., L.G. Meira Filho, B.A. Callander, N. Harris, A. Kattenberg and K. Maskell, 1996: Climate Change 1995, The Science of Climate Change. Cambridge: Cambridge University Press, $527 \mathrm{pp}$.

Koscielny-Bunde, E., H.E. Roman, A. Bunde, S. Havlin, H.J. Schellnhuber, 1998: Longrange power-law correlations in local daily temperature fluctuations. Phil. Mag. B, 77, 1331-1340.

Mann, M.E. and J. Park, 1994: Global-scale modes of surface temperature variability on interannual to century timescales. J. Geoph. Res., 99, D12, 25,819-25,833.

Mann, M.E. and J. Park, 1996: Greenhouse warming and changes in the seasonal cycle of temperature: Model versus observations. Geoph. Res. Let., 23, $1111-1114$.

Moron, V., R. Vautard, and M. Ghil, 1998: Trends, interdecadal and interannual oscillations in global sea-surface temperatures. Climate Dynamics, 14, 545 - 569.

Nicholls, N., G.V. Gruza, J. Jouzel, T.R. Karl, L.A. Ogallo, and D.E. Parker, 1996: Observed Climate Variability and Change, in: Houghton, J.T., L.G. Meira Filho, B.A. Callander, N. Harris, A. Kattenberg and K. Maskell, 1996: Climate Change 1995, The Science of Climate Change. Cambridge: Cambridge University Press, 527 pp.

Parker, D.E., P.D. Jones, C.K. Folland, and A. Bevan, 1994: Interdecadal changes of surface temperature since the late nineteenth century. J. Geoph. Res., 99, 14373 - 14399. 
Plaut, G., M. Ghil and R. Vautard, 1995: Interannual and Interdecadal Variability in 335 Years of Central England Temperatures. Science, 268, 710 - 713.

Schlesinger, M.E. and N. Ramankutty, 1994: An oscillation in the global climate system of period $65-70$ years. Nature, 367, $723-726$.

Schlesinger, M.E. and N. Ramankutty, 1994: Reply to Elsner and Tsonis, 1994. Nature, 372, $508-509$.

Schönwiese, C.-D. and J. Rapp, 1997: Climate Trend Atlas of Europe Based on Observations 1891 - 1990. Dordrecht, Kluwer Academic Publishers, 228 pp.

Thompson, D.J., 1995: The Seasons, Global Temperature, and Precession. Science, 268, $59-68$.

Tsonis, A.A. and J.B. Elsner, 1992: Oscillating global temperature. Nature, 356, 751.

Tsonis, A.A., P.J. Roebber and J.B. Elsner, 1998: A characteristic time scale in the global temperature record. Geoph. Res. Let., 25, $2821-2823$.

Vautard, R. and M. Ghil, 1989: Singular Spectrum Analysis in nonlinear dynamics with applications to paleoclimatic time series. Physica D, 35, 395-424.

Vislocky, R. V. and J. M. Fritsch, 1995: Generalized Additive Models versus Linear Regression in Generating Probabilistic MOS Forecasts of Aviation Weather Parameters. Weather and Forecasting, 10, 669-680.

von Storch, H. and F.W. Zwiers, 1999: Statistical Analysis in Climate Research. Cambridge, Cambridge University Press, 484 pp.

Werner, A., 1999: Die Nord-Atlantik-Oszillation und ihre Auswirkungen auf Europa. Frankfurt: Diploma Thesis, Inst. for Meteorology and Geophysics, University Frankfurt, 91 pp.

Zheng, X. and R. E. Basher, 1998: Structural Time Series Models and Trend Detection in Global and Regional Temperature Series. J. Clim., 12, 2347-2357. 